\begin{tabular}{|c|l|}
\hline Title & Topological classification of the tangent developables of space curves \\
\hline Author(s) & Ishikawa, G. \\
\hline Citation & Hokkaido University Preprint Series in Mathematics, 341, 1-16 \\
\hline Issue Date & 1996-7-1 \\
\hline DOI & 10.14943/83487 \\
\hline Doc URL & http://hdl.handle.net/2115/69091 \\
\hline Type & bulletin (article) \\
\hline File Information & pre341.pdf \\
\hline
\end{tabular}

Instructions for use 
Topological Classification of the Tangent Developables of Space Curves

\section{Goo Ishikawa}

Series \#341. July 1996 


\section{HOKKAIDO UNIVERSITY PREPRINT SERIES IN MATHEMATICS}

\#317 M.-H. Giga and Y. Giga, Consistency in evolutions by crystalline curvature, 16 pages. 1995.

\#318 Wei-Zhi Sun, Shadows of moving surfaces, 19 pages. 1995.

\#319 S. Izumiya and G.T. Kossioris, Bifurcations of shock waves for viscosity solutions of Hamilton-Jacobi equations of one space variable, 39 pages. 1995.

\#320 T. Teruya, Normal intermediate subfactors, 44 pages. 1995.

\#321 M. Ohnuma, Axisymmetric solutions and singular parabolic equations in the theory of viscosity solutions, 26 pages. 1995.

\#322 T. Nakazi, An outer function and several important functions in two variables, 12 pages. 1995.

\#323 N. Kawazumi, An infinitesimal approach to the stable cohomology of the moduli of Riemann surfaces, 22 pages. 1995.

\#324 A. Arai, Factorization of self-adjoint operators by abstract Dirac operators and its application to second quantizations on Boson Fermion Fock spaces, 15 pages. 1995.

\#325 K. Sugano, On strongly separable Frobenius extensions, 11 pages. 1995.

\#326 D. Lehmann and T. Suwa, Residues of holomorphic vector fields on singular varieties, 21 pages. 1995.

\#327 K. Tsutaya, Local regularity of non-resonant nonlinear wave equations, 23 pages. 1996.

\#328 T. Ozawa and Y. Tsutsumi, Space-time estimates for null gauge forms and nonlinear Schrödinger equations, 25 pages. 1996.

\#329 O. Ogurisu, Anticommutativity and spin 1/2 Schrödinger operators with magnetic fields, 12 pages. 1996.

\#330 Y. Kurokawa, Singularities for projections of contour lines of surfaces onto planes, 24 pages. 1996.

\#331 M.-H. Giga and Y. Giga, Evolving graphs by singular weighted curvature, 94 pages. 1996.

\#332 M. Ohnuma and K. Sato, Singular degenerate parabolic equations with applications to the $p$-laplace diffusion equation, 20 pages. 1996.

\#333 T. Nakazi, The spectra of Toeplitz operators with unimodular symbols, 9 pages. 1996.

\#334 B. Khanedani and T. Suwa, First variation of horomorphic forms and some applications, 11 pages. 1996.

\#335 J. Seade and T. Suwa, Residues and topological invariants of singular holomorphic foliations ${ }^{1}, 28$ pages. 1996.

\#336 Y. Giga, M.E. Gurtin and J. Matias, On the dynamics of crystalline motions, 67 pages. 1996.

\#337 I. Tsuda, A new type of self-organization associated with chaotic dynamics in neural networks, 22 pages. 1996.

\#338 F. Hiroshima, A scaling limit of a Hamiltonian of many nonrelativistic particles interacting with a quantized radiation field, 34 pages. 1996.

\#339 N. Tominaga, Analysis of a family of strongly commuting self-adjoint operators with applications to perturbed Dirac operators, 29 pages. 1996.

\#340 A. Inoue, Abel-Tauber theorems for Fourier-Stieltjes coefficients, 17 pages. 1996. 


\title{
Topological Classification of the Tangent Developables of Space Curves
}

\author{
by Goo ISHIKAWA
}

July 15, 1996

\section{Introduction}

The tangent lines to a space curve form a ruled surface, which is called the tangent developable of the space curve. In the present paper we give the local topological classification of the tangent developables of space curves.

In the classical differential geometry, the tangent developables appear as surfaces of zero curvature (cf. [23]). Also in the classical algebraic geometry, they play a significant role in the duality theory of space curves (cf. [6]). Therefore they were well known objects, in particular in the last century. The complete description of their structure, however, involves the singularity theory developed in this century, as we can see in [5][20], for instance.

First recall the notions and results used in this paper on the singularities of the tangent developables of space curves from [21],[11].

Let $\gamma:\left(\mathbf{R}, t_{0}\right) \rightarrow \mathbf{R}^{3}$ be a germ of $C^{\infty}$ parametrised space curve at a $t_{0} \in \mathbf{R}$. Representing $\gamma$ as $\boldsymbol{x}(t)=\left(x_{1}(t), x_{2}(t), x_{3}(t)\right)$, we call $\gamma$ of finite type at $t_{0}$ if the infinite number of vectors $\dot{\boldsymbol{x}}\left(t_{0}\right), \ddot{\boldsymbol{x}}\left(t_{0}\right), \cdots, \boldsymbol{x}^{(k)}\left(t_{0}\right), \cdots$, generate the three dimensional space. Then, for some affine coordinates and for some positive integers $m, s, r$, the curve $\gamma$ is written under the form

$$
x_{1}(t)=t^{m}+o\left(t^{m}\right), x_{2}(t)=t^{m+s}+o\left(t^{m+s}\right), x_{3}(t)=t^{m+s+r}+o\left(t^{m+s+r}\right) .
$$

The triplet $(m, m+s, m+s+r)$ is in fact independent of the choice of affine local coordinates, and it is called the type of the curve-germ $\gamma$ : type $(\gamma)=\left(a_{1}, a_{2}, a_{3}\right)=(m, m+$ $s, m+s+r)$. Notice that, a curve-germ is of finite type if the curve does not have infinite tangency with any affine plane.

We treat here only curve-germs of finite type, excluding plane curves essentially. Notice that, in a generic multi-dimensional family of curves, there appear only curve-germs of finite type ([21] Theorem 2.2). 
We call a point $t_{0}$ an ordinary point where type $(\gamma)$ at $t_{0}$ is equal to $(1,2,3)$. Otherwise we call it a special point. Special points are isolated. The curve is nonsingular at $t_{0}$ if and only if $a_{1}=m=1$.

The tangent developable of a curve is defined as the union of tangent lines to the curve. Here the tangent line at a singular point is defined as the limit of tangent lines at non-singualr points. In a neighborhood of a non-singular point, the tangent developable has the parametrisation $(t, s) \mapsto \boldsymbol{x}(t)+s \dot{\boldsymbol{x}}(t)$.

At an ordinary point, it is classically known that the tangent developable has the cuspidal edge along the curve itself. (See [5][20].) For the simplest case of special points, namely in the case of type $(1,2,4)$, recently Cleave [7] gave the $C^{\infty}$ normal form of the tangent developables: The surface has, besides the cuspidal edge, a self-intersection locus leaving from the special point. (See also [8] for another approach in this case.) Then Mond [18],[19] proceeded to the $C^{\infty}$ classification of tangent developables at several more degenerate special points.

Meanwhile, Shcherbak [21] applied Legendre singularity theory to the study of singularities of tangent developables via the projective duality. The tangent developable of a space curve is a ruled surface and tangent planes to regular points of the surface are constant along each generating line: They are called osculating planes. The osculating planes to a space curve of type $(m, m+s, m+s+r)$ form a space curve in the dual projective space of type $(r, r+s, r+s+m)$; the dual curve [21]. Then the tangent developable of a curve is the projection to the original space of the projective conormal bundle over the dual curve in the dual projective space.

From the general framework it is given the parametrisation of the tangent developable near a possibly singular point of the curve, which covers also the non-singular case. Let $t_{0}=0$. The tangent developable of a curve of type $(m, m+s, m+s+r)$ has a parametrisation $f:\left(\mathbf{R}^{2}, 0\right) \rightarrow\left(\mathbf{R}^{3}, 0\right)$ defined by

$$
\begin{aligned}
x_{1} & =x, \\
x_{2} & =t^{s+m}+t^{s+m+1} \varphi(t)+x\left(t^{s}+t^{s+1} \psi(t)\right), \\
x_{3} & =(r+s)(r+s+m) \int_{0}^{t} u^{r} \frac{\partial x_{2}(u, x)}{\partial u} d u \\
& =(r+s)(s+m) t^{r+s+m}+\cdots+x\left\{s(r+s+m) t^{r+s}+\cdots\right\},
\end{aligned}
$$

for some $C^{\infty}$ functions-germs $\varphi(t)$ and $\psi(t)$. (See $\S 2$.)

To classify the singularities arising from the tangent developables, thus we are naturally led to the problem about the determinacy of the parametrisations by the leading terms. Remark that we are treating very degenerate singularities even in the sense of Legendre singularity theory, because the singularities along $\{t=0\}$ are more degenerate than the ordinary cuspidal edge if $s \geq 3$ or $r \geq 2$.

Then, using the infinitesimal calculation, we overcame this difficulty and showed in [11][13] about the differentiable classification as follows: The local diffeomorphism class of 
the tangent developable is determined by the type $\mathbf{A}$ of a space curve if and only if $\mathbf{A}$ is one of the followings: $(1,2,2+r), r$ : positive integer, $(2,3,4),(1,3,4),(3,4,5),(1,3,5)$. This result indicates the necessity, in the differentiable classification, of "secondary invariants" of a curve than just the type to classify more degenerate cases.

The above result also turns our concern to the topological classification of tangent developables, since we naturally suppose that the topological classification will be achieved for wider classes of curves and in a simpler manner than the differentiable classification.

As for the topological classification, Mond [19] proved that the tangent developable of a curve of type $(1,2,2+r), r=1,2, \ldots$, is homeomorphic to the plane if $r$ is odd and to the cone of the figure eight on the sphere if $r$ is even. Nevertheless, no result is known succeeding Mond's result about the local topological classification of the tangent developables of space curves, as far as the author knows.

Then the purpose of this paper is to prove the following topological classification theorem:

Theorem 1.1 The local homeomorphism class of the tangent developable of a space curve is determined by the type $(m, m+s, m+s+r)$ if and only if $s, r$ are not both even, or, $m=1$ and $s, r$ are both even. Actually, in this case, the homeomorphism class is determined by the parities of $(m, s, r)$ : The tangent developable is homeomorphic to the cone of the spherical curve of Fig. 1 in each case.

See Figure 2 for the illustration of the tangent developable in each case.

In particular we see, from Theorem 1.1, the following:

Corollary 1.2 The homeomorphism class of the tangent developable of a non-singular space curve is determined by the type $(1,1+s, 1+s+r)$.

The "only if" part of Theorem 1.1 is a consequence of the following more exact result:

Proposition 1.3 If $m$ is odd, $m \geq 3$ and $s, r$ are both even, then there exist exactly two homeomorphism classes for the tangent developables of curves of type $(m, m+s, m+s+r)$. If $m, s$ and $r$ are all even, then there exist uncountably many homeomorphism classes, for each type $(m, m+s, m+s+r)$.

In the process of proving Theorem 1.1 we find out general local properties for the tangent developables.

Proposition 1.4 If $m, s, r$ are not even, then the tangent developable of a curve of type $(m, m+s, m+s+r)$ has, near the base point,

(1) no double points on the curve itself and on the tangent line at the base point, 
(2) no points of self-tangency,

(3) no triple points, and

(4) a topological cone structure.

In the complex analytic category, in the similar manner, we can define the tangent developable $\left(\mathbf{C}^{2}, 0\right) \rightarrow\left(\mathbf{C}^{3}, 0\right)$ of a holomorphic space curve $(\mathbf{C}, 0) \rightarrow\left(\mathbf{C}^{3}, 0\right)$. The complex analytic classification theorem then holds exactly same as in the real differentiable classification [12]. However, even for a non-singular curve, the homeomorphism class of a developable over the complex is not determined by the type of the curve in general. The essential point is that the above property (3) does not holds for a complex analytic curve. For instance, consider the non-singular curve $(\mathbf{C}, 0) \rightarrow\left(\mathbf{C}^{3}, 0\right)$

$$
x_{1}(t)=t, x_{2}(t)=t^{3}, x_{3}(t)=t^{6} \text {. }
$$

It has $\mathbf{Z} / 3 \mathbf{Z}$ symmetry under the linear mapping $\left(x_{1}, x_{2}, x_{3}\right) \mapsto\left(\zeta x_{1}, x_{2}, x_{3}\right), \zeta^{3}=1, \zeta \neq 1$, fixing the plane $\left\{x_{1}=0\right\}$ pointwise, and under the re-parametrisation $t \mapsto \zeta t$. Therefore, on the plane $\left\{x_{1}=0\right\}$ and near $t_{0}=0$, this curve has a locus of points from which there are three tangent lines to the curve, that is, the triple points locus of the tangent developable. Remark that the type $(1,3,6)$ lies outside of the class of types such that each type determines the holomorphic isomorphism class of the tangent developables [12], [13].

Even in the real case, if $m, s$ and $r$ are all even, then, by Theorem 1.1, the topological type of the tangent developable depends on the secondary or more higher terms of the original curve. Though the general formulation is beyond the scope of this paper, the philosophy of finite determinacy, then, should be crucial to this classification problem as in the usual singularity theory [24].

The tangent developable appears also as a caustic. Consider the generating family $G=t^{6}+x_{1} t^{4}+x_{2} t^{2}+x_{3} t$, which has Z/2Z-symmetry $\left(t ; x_{1}, x_{2}, x_{3}\right) \mapsto\left(-t ; x_{1}, x_{2},-x_{3}\right)$ and is quasi-homogeneous with respect to weight $\left(t ; x_{1}, x_{2}, x_{3}\right)=(1 ; 2,4,5)$. The caustic

$$
\left\{\left(x_{1}, x_{2}, x_{3}\right) \in\left(\mathbf{R}^{3}, 0\right) \mid \partial G / \partial t=\partial^{2} G / \partial^{2} t=0 \text { for some } t\right\}
$$

is called the symmetric butterfly and stable under symmetric perturbations of $G$ [14]. We remark it is also a tangent developable of a curve of type $(2,4,5)$. Therefore, as an application of Theorem 1.1, we see the following:

Corollary 1.5 Under a higher order but not necessarily symmetric perturbations

$$
\tilde{G}=\left(t^{6}+a(t)\right)+x_{1}\left(t^{4}+b(t)\right)+x_{2}\left(t^{2}+c(t)\right)+x_{3}(t+d(t)),
$$

$\operatorname{ord}(a) \geq 7, \operatorname{ord}(b) \geq 5, \operatorname{ord}(c) \geq 3, \operatorname{ord}(d) \geq 2$, the caustics are all homeomorphic to the symmetric butterfly. 
We remark that also the global topology of the tangent developables is investigated recently by many authors: See, for instance, [3].

Remark that it is treated in [21][22][11][12][13] the differentiable classification of developable hypersurfaces of curves in arbitrary dimensional ambient spaces. The essentially new ideas, however, will be required for the corresponding topological classification in higher dimensional cases: Our method in this paper is limited to the space curves.

In the next section we give parametrisations of the tangent developables of space curve-germs. The map-germs thus obtained, form a restricted class of heavily degenerate singularities, which is the exact object in the present paper.

In $\S 3$, we study the tangent developable of a typical curve for each type. Actually we give a stratification of the parametrisation, examining the properties of Proposition 1.4. Then we know that the "fewnomial phenomena" (cf. [15]) manifests as the small variations of homeomorphism classes for the tangent developables, while the "determinacy phenomena" by leading terms concludes the classification.

$\S 4$ is devoted to prove the main part of Theorem 1.1. We observe that, if the type $(m, m+s, m+s+r)$ satisfies the assumption of Theorem 1.1, then the stratification of the tangent developable behaves well under a type-preserving deformation of the curve. Then we see Proposition 1.4 uniformly, as well as Whitney regularity of the stratification to prove the topological triviality of the family. The essential ingredient is the Kuiper-Kuo theorem [16][17]: This is used to guarantee the non-existence of bifurcations of double points loci.

In the last section we prove Proposition 1.3 and complete the proof of Theorem 1.1.

The author presents his gratitudes to Professors S. Izumiya, S. Koike, T. Morimoto, I. Nakai, T. Ozawa, M.J. Saia and C.T.C. Wall for valuable suggestion and encouragement.

\section{Parametrisation of a tangent developable}

Let $\gamma:(\mathbf{R}, 0) \rightarrow \mathbf{R} P^{3}$ be a curve-germ of type $(m, m+s, m+s+r)$. Then the dual $\gamma^{*}:(\mathbf{R}, 0) \rightarrow \mathbf{R} P^{3 *}$ of $\gamma$ in the dual projective space $\mathbf{R} P^{3 *}$ is of type $(r, r+s, r+s+m)$ [21]. In fact this can be seen directly by solving

$$
\begin{array}{cc}
x_{3}(t)+x_{2}(t) y_{1}+x_{1}(t) y_{2}+y_{3} & =0 \\
\dot{x}_{3}(t)+\dot{x}_{2}(t) y_{1}+\dot{x}_{1}(t) y_{2} & =0 \\
\ddot{x}_{3}(t)+\ddot{x}_{2}(t) y_{1}+\ddot{x}_{1}(t) y_{2} & =0 .
\end{array}
$$


Then fix nonzero constants $a, b, c$, and set

$$
\gamma^{*}: y_{1}=a t^{r}+o\left(t^{r}\right), y_{2}=b t^{r+s}+o\left(t^{r+s}\right), y_{3}=c t^{r+s+m}+o\left(t^{r+s+m}\right)
$$

for some affine coordinates of $\left(\mathbf{R} P^{3 *}, \gamma^{*}(0)\right)$. By a change of $C^{\infty}$ coordinate of $(\mathbf{R}, 0)$, we delete the higher order terms of $y_{1}$. Set

$$
F(t, x)=y_{3}(t)+y_{2}(t) x_{1}+y_{1}(t) x_{2}+x_{3} .
$$

Then we have a parametrisation of the tangent developable of $\gamma$ (resp. the curve $\gamma$ itself), solving the equation $F=\partial F / \partial t=0$ (resp. $F=\partial F / \partial t=\partial^{2} F / \partial t^{2}=0$ ), first for $t \neq 0$, and then extending the solution to $t=0$. If we set further $a=-(r+s)(r+s+m), b=$ $-r(r+s+m), c=-r(r+s)$, just for simplifying the expression, we get the parametrisation $f$ given in $\S 1$.

The map-germ $f$ is not immersive along the locus $\left\{(t, x) \mid \partial x_{2} / \partial t=0\right\}$ in $\left(\mathbf{R}^{2}, 0\right)$. Therefore the tangent developable is singular along $\gamma$ parametrised by a curve $C_{0}(f)=$ $\left\{x=-((s+m) / s) t^{m}+o\left(t^{m}\right)\right\}$, and, in the case $s>1$, also along the tangent line to the base point parametrised by $L=\{(t, x) \mid t=0\}$. Further we set

$$
M(f)=\text { the closure of }\left\{(t, x) \mid \# f^{-1}(f(t, x)) \geq 2\right\}
$$

$D_{0}(f)=f\left(C_{0}(f)\right), L^{\prime}=f(L)=\left\{x_{2}=x_{3}=0\right\}$ and $W(f)=f(M(f))$. Following sections are mainly devoted to clarifying the relative positions of $M(f), C_{0}(f)$ and $L$ (resp. $W(f), D_{0}(f)$ and $L^{\prime}$ ).

We remark that the construction above is valid also for a $C^{\infty}$ family $\gamma_{\lambda},(\lambda \in \Lambda)$, of curve-germs of constant type $(m, m+s, m+s+r)$ : We have a $C^{\infty}$ family of $C^{\infty}$ coordinates of $(\mathbf{R}, 0)$, and of affine coordinates of $\left(\mathbf{R}^{3}, \gamma_{\lambda}(0)\right)$ such that $\gamma_{\lambda}$ is represented by

$$
x_{1}=t^{m}+t^{m+1} P(t, \lambda), \quad x_{2}=t^{m+s}+t^{m+s+1} Q(t, \lambda), \quad x_{3}=t^{m+s+r}+t^{m+s+r+1} R(t, \lambda),
$$

for $C^{\infty}$ function-germs $P, Q, R:(\mathbf{R} \times \Lambda, 0 \times \Lambda) \rightarrow \mathbf{R}$. Thus we see as above that the duals $\gamma_{\lambda}^{*}$ form a $C^{\infty}$ family $\left(y_{1}(t, \lambda), y_{2}(t, \lambda), y_{3}(t, \lambda)\right)$ of constant type $(r, r+s, r+s+m)$. Remark that, for a $C^{\infty}$ family $\gamma_{\lambda}$ which is not necessarily type-preserving, the duals $\gamma_{\lambda}$ do not depend smoothly on $\lambda$ in general.

Now the family of the tangent developables of $\gamma_{\lambda}$ has a uniform parametrisation $f_{\lambda}$ : $\left(\mathbf{R}^{2}, 0\right) \rightarrow\left(\mathbf{R}^{3}, 0\right)$ of the form

$$
x_{1}=x, \quad x_{2}=B+x A, \quad x_{3}=(r+s)(r+s+m) \int_{0}^{t} u^{r} \frac{\partial x_{2}(u, x, \lambda)}{\partial u} d u=B^{\prime}+x A^{\prime},
$$

with

$$
\begin{gathered}
B(t, \lambda)=t^{s+m}+t^{s+m+1} \varphi(t, \lambda), A(t, \lambda)=t^{s}+t^{s+1} \psi(t, \lambda), \\
B^{\prime}(t, \lambda)=(r+s)(s+m) t^{r+s+m}+t^{r+s+m+1} \tilde{\varphi}(t, \lambda),
\end{gathered}
$$




$$
A^{\prime}(t, \lambda)=s(r+s+m) t^{r+s}+t^{r+s+1} \tilde{\psi}(t, \lambda)
$$

for some $C^{\infty}$ functions $\varphi$ and $\psi$, where $\tilde{\varphi}$ and $\tilde{\psi}$ are determined by $\varphi$ and $\psi$ respectively. Conversely, for any $C^{\infty}$ function-germs $\varphi$ and $\psi:(\mathbf{R} \times \Lambda, 0 \times \Lambda) \rightarrow \mathbf{R}$, the family of map-germs $f_{\lambda}$ represents a uniform parametrisation of tangent developables for a family of curves of constant type $(m, m+s, m+s+r)$.

Moreover $f_{\lambda}$ lifts to $\tilde{f}_{\lambda}:\left(\mathbf{R}^{2}, 0\right) \rightarrow \mathbf{R} P^{3} \times \mathbf{R} P^{3 *}$, by

$$
(t, x) \mapsto\left(x_{1}(t, x, \lambda), x_{2}(t, x, \lambda), x_{3}(t, x, \lambda) ; y_{1}(t, \lambda), y_{2}(t, \lambda), y_{3}(t, \lambda)\right),
$$

Then the family $\tilde{f}_{\lambda}$ satisfies $y_{3}+y_{2} x_{1}+y_{1} x_{2}+x_{3}=0$, projects to the family of dual curves $\gamma_{\lambda}^{*}$ and therefore describes the behaviour of tangent spaces to the developables. We call $\tilde{f}_{\lambda}$ the Nash lifting of $f_{\lambda}[12]$.

If we set

$$
F=\left(f_{\lambda}, \lambda\right):\left(\mathbf{R}^{2} \times \Lambda, 0 \times \Lambda\right) \rightarrow\left(\mathbf{R}^{3} \times \Lambda, 0 \times \Lambda\right),
$$

then we have a $C^{\infty}$ map

$$
\tilde{F}=\left(\tilde{f}_{\lambda}, \lambda\right):\left(\mathbf{R}^{2} \times \Lambda, 0 \times \Lambda\right) \rightarrow \mathbf{R} P^{3} \times \mathbf{R} P^{3 *} \times \Lambda,
$$

which we call the Nash lifting of the unfolding $F$.

\section{Analysis on unperturbed cases}

Consider the map-germ $f_{0}:\left(\mathbf{R}^{2}, 0\right) \rightarrow\left(\mathbf{R}^{3}, 0\right)$ defined by leading terms:

$$
f_{0}(t, x)=\left(x, t^{s+m}+x t^{s},(r+s)(s+m) t^{r+s+m}+s(r+s+m) x t^{r+s}\right)
$$

of $f$. The map-germ $f_{0}$ is a parametrisation of the tangent developable of a curve the dual of which is defined by

$$
y_{1}=-(r+s)(r+s+m) t^{r}, y_{2}=-r(r+s+m) t^{r+s}, y_{3}=-r(r+s) t^{r+s+m} .
$$

Now we examine the set consisting of points $(t, x)$ with $\# f_{0}^{-1}\left(f_{0}(t, x)\right) \geq 2$.

Let two points $\left(t_{1}, x\right)$ and $\left(t_{2}, x\right)$ in $\mathbf{R}^{2}$ have same image by $f_{0}$. Then

$$
\left(\begin{array}{cc}
t_{2}^{s}-t_{1}^{s}, & t_{2}^{s+m}-t_{1}^{s+m} \\
s(r+s+m)\left(t_{2}^{r+s}-t_{1}^{r+s}\right), & (r+s)(s+m)\left(t_{2}^{r+s+m}-t_{1}^{r+s+m}\right)
\end{array}\right)\left(\begin{array}{l}
x \\
1
\end{array}\right)=\left(\begin{array}{l}
0 \\
0
\end{array}\right),
$$

and therefore the determinant

$$
d_{0}\left(t_{1}, t_{2}\right)=\left|\begin{array}{cc}
t_{2}^{s}-t_{1}^{s}, & t_{2}^{s+m}-t_{1}^{s+m} \\
s(r+s+m)\left(t_{2}^{r+s}-t_{1}^{r+s}\right), & (r+s)(s+m)\left(t_{2}^{r+s+m}-t_{1}^{r+s+m}\right)
\end{array}\right|
$$


must vanish for such $t_{1}$ and $t_{2}$. To investigate the zero set of $d_{0}$ on the $\left(t_{1}, t_{2}\right)$ plane, set

$$
\begin{aligned}
g(z)= & (1 / r m) d_{0}(1, z) \\
= & z^{r+2 s+m}-\frac{(r+s)(s+m)}{r m} z^{r+s+m}+\frac{s(r+s+m)}{r m} z^{r+s} \\
& +\frac{s(r+s+m)}{r m} z^{s+m}-\frac{(r+s)(s+m)}{r m} z^{s}+1 .
\end{aligned}
$$

Then we observe the followings which are easily verified:

Lemma 3.1 (0) $z=\xi$ is a real root of $g(z)=0$ if and only if $t_{2}=\xi t_{1}$ is a real branch of $\left\{d_{0}\left(t_{1}, t_{2}\right)=0\right\}$.

(1) $z=1$ is a multiple root of $g(z)=0$ of multiplicity 2 .

(2) If $\xi$ is a root, then also $1 / \xi$ is a root of $g(z)=0$.

(3) If there exist a triple point of $f_{0}$ then there exist non-trivial $(\neq 1)$ roots $\xi, \eta$ of $g(z)=0$ such that the product $\xi \eta$ is also a non-trivial root of $g(z)=0$.

Now we naturally number the parities of $(m, s, r)$ that we treat as follows: (i): $m$ is odd, $s$ is odd and $r$ is odd, (ii): $m$ is odd, $s$ is odd and $r$ is even, (iii): $m$ is odd, $s$ is even and $r$ is odd, (iv): $m$ is odd, $s$ is even and $r$ is even, (v): $m$ is even, $s$ is odd and $r$ is odd, (vi): $m$ is even, $s$ is odd and $r$ is even, (vii): $m$ is even, $s$ is even and $r$ is odd.

Lemma 3.2 The real roots other than 1 of the equation $g(z)=0$ are all negative and simple in all seven cases. In each case, the number of them is equal to (i): 0, (ii): 1, (iii): 2, (iv): 3, (v): 1, (vi): 0, (vii): 3 . In particular, -1 is a root of $g(z)=0$ just in the cases (ii), (iv), (v) and (vii).

Proof: To study the real zeros of $g(z)$ we recall the famous theorem of Descartes: The number of real positive roots of real algebraic equation $g=0$, counted with multiplicities, is at most the number of sign-changes of terms of $g$.

Then we see $g(z)=0$ has at most 4 positive real roots. Since 1 is the multiple root, $g^{\prime \prime}(1)>0, \lim _{z \rightarrow \infty} g(z)=\infty, g(0)=1$, we see, by Lemma 3.1.(2), $g(z)=0$ has no real positive root other than $z=1$.

For negative roots, we apply the theorem of Descartes to $g(-z)$, and check up all seven classes. For instance, for the case (vii), the number of sign-changes is equal to 3 if $r>m$, and 5 if $r<m$. Further we see $g(-1)=0,(d g / d z)(-1)=-2(s+m) s / r<0$ and $\lim _{z \rightarrow-\infty} g(z)=-\infty$. Therefore by Lemma 3.1.(2), we see there exist just 3 negative roots. The arguments for remaining cases are similar and we omit them.

Remark 3.3 : For the case (viii) : $m, s$ and $r$ are all even, the polynomial $G(z)$ has -1 as a multiple root. This implies essentially the indeterminacy of the topological type of $f$ in this case. 
Now the zero set $d_{0}^{-1}(0)$ consists of lines $L_{\xi}: t_{2}=\xi t_{1}, \xi$ being real roots of $g(z)=0$.

Let $\xi \neq 1,-1$ and $\left(t_{1}, t_{2}\right) \in L_{\xi}$ with $t_{1} \neq 0$. Then we have

$$
x=-\left(t_{2}^{s+m}-t_{1}^{s+m}\right) /\left(t_{2}^{s}-t_{1}^{s}\right)=\eta t_{1}^{m},
$$

setting $\eta=-\left(\xi^{s+m}-1\right) /\left(\xi^{s}-1\right)$. Therefore, on the $(t, x)$-plane, we have, as components of $M\left(f_{0}\right), C_{\xi}: x=\eta t^{m}$ for $\xi$, and, $C_{1 / \xi}: x=\left(\eta / \xi^{m}\right) t^{m}$ for $1 / \xi$. Moreover we see, for $\left(t, \eta t^{m}\right) \in C_{\xi}$, that $\left(t,\left(\eta / \xi^{m}\right) t^{m}\right) \in C_{1 / \xi}$, and that $f_{0}\left(t, \eta t^{m}\right)=f_{0}\left(t,\left(\eta / \xi^{m}\right) t^{m}\right)$.

Let $\xi=-1$ and consider the line $L_{-1}: t_{2}=-t_{1}$. By Lemma 3.2, we face the cases (ii), (iv), (v) and (vii). In the case (iv), there does not exist $x$ with $f_{0}(t, x)=f_{0}(-t, x)$, for any $t \neq 0$. For the case (ii) (resp. (v), (vii)), we have, as one of components of $M\left(f_{0}\right)$, $C_{-1}: x=0$, (resp. $\left.x=-t^{m}, x=-\{(r+s)(s+m) / s(r+s+m)\} t^{m}\right)$, which is mapped by $f_{0}$ two to one, outside of the origin.

Thus we see that $M\left(f_{0}\right)=C_{\xi} \cup C_{1 / \xi} \cup C_{-1}$ in the case (vii), $M\left(f_{0}\right)=C_{\xi} \cup C_{1 / \xi}$ in the cases (iii) and (iv), $M\left(f_{0}\right)=C_{-1}$ in the cases (ii) and (v), and that $M\left(f_{0}\right)=\{(0,0)\}$ in the cases (i) and (vi).

Notice that the Nash lifting $\tilde{f}_{0}$ separates each pair of double point locus on the $(t, x)$ plane, and therefore, $f_{0}$ has no self-tangent points.

The curve $C_{0}\left(f_{0}\right)=\left\{x=-((s+m) / s) t^{m}\right\}$ parametrising the original curve is obtained as $\left\{(t, x) \mid \partial x_{2} / \partial t=0, t \neq 0\right\} \cup\{(0,0)\}$. The line $L=\{t=0\}$ corresponds to the tangent line to the original curve at the base point.

To determine the homeomorphism class of $f_{0}$ in each of seven cases, we need to know the relative position, in the $(t, x)$ plane, between the branches of $C_{\xi}, C_{1 / \xi}, C_{-1}, C_{0}$ and $L$. For instance, for the case (vii), we verify that

$$
\eta<-\frac{s+m}{s}<-\frac{(r+s)(s+m)}{s(r+s+m)}<\frac{\eta}{\xi^{m}}<0,
$$

for $\xi<-1<1 / \xi$ and $\eta=-\left(\xi^{s+m}-1\right) /\left(\xi^{s}-1\right)$, using the Cauchy's mean value theorem.

Then we get the configuration of double point loci and singular loci to describe the topological types of $f_{0}$ in each case: Figure 3. They are regarded as the Gauss diagrams ([2]) for the link mapping $S^{1} \rightarrow S^{2}$ of the map-germ $f_{0}$ in an extended sense.

It is helpful, when we write down the pictures of Figure 2, to remark that

$$
\left(\partial x_{3} / \partial t\right) /\left(\partial x_{2} / \partial t\right)=t^{r}
$$

and that therefore the tangent lines to the slices of developables by planes $\{x=$ const. $\}$ behave simply.

Set $h_{0}\left(t_{1}, t_{2}\right)=\left(t_{1}-t_{2}\right)^{-2} d_{0}\left(t_{1}, t_{2}\right)$. Then the following result is necessary in $\S 4$, which is, via Lemma 3.2, the direct consequence of Kuiper-Kuo theorem [16][17]: 
Lemma 3.4 The homogeneous polynomial $h_{0}:\left(\mathbf{R}^{2}, 0\right) \rightarrow(\mathbf{R}, 0)$ of degree $r+2 s+m-2$ has the isolated singularity at the origin and is $(r+2 s+m-2)$-determined with respect to the $C^{1}-V$ equivalence of $C^{\infty}$ map-germs. More strictly, for any $C^{\infty}$ family $h_{\lambda}:\left(\mathbf{R}^{2}, 0\right) \rightarrow$ $(\mathbf{R}, 0), \lambda \in \Lambda$, with the same $(r+2 s+m-2)$-jets as $h_{0}$, the zero-curves $h_{\lambda}^{-1}(0)$ are locally trivialised by a family of $C^{1}$ diffeomorphisms $\sigma_{\lambda}$ with the identity linear parts: $\sigma_{\lambda}\left(h_{\lambda}^{-1}(0)\right)=h_{0}^{-1}(0)$ on a neighborhood of 0 , for any $\lambda$ on a compact set of $\Lambda$.

\section{Topological triviality}

In this section we will show the following, which implies the "if" part of Theorem 1.1.

Proposition 4.1 If $s$ and $r$ are not both even, or, $m=1$ and $s, r$ are both even, then any family $f_{\lambda}$ of parametrisations of tangent developables of curves of constant type $(m, m+$ $s, m+s+r)$ is locally topologically trivial.

Let $f:\left(\mathbf{R}^{2}, 0\right) \rightarrow\left(\mathbf{R}^{3}, 0\right)$ be the $C^{\infty}$ parametrisation of the tangent developable of a space curve of type $(m, m+s, m+s+r)$ as in $\S 1$. To prove the "if" part of Theorem 1.1, we have to show $f_{0}$ and $f$ are topologically equivalent, that is, there exist homeomorphismgerms $\sigma:\left(\mathbf{R}^{2}, 0\right) \rightarrow\left(\mathbf{R}^{2}, 0\right)$ and $\tau:\left(\mathbf{R}^{3}, 0\right) \rightarrow\left(\mathbf{R}^{3}, 0\right)$ such that $f \circ \sigma=\tau \circ f_{0}$.

Set

$$
F(t, x, \lambda)=\left((1-\lambda) f_{0}(t, x)+\lambda f(t, x), \lambda\right):\left(\mathbf{R}^{2} \times \Lambda, 0 \times \Lambda\right) \rightarrow\left(\mathbf{R}^{3} \times \Lambda, 0 \times \Lambda\right),
$$

for an open interval $\Lambda$ containing $I=[0,1]$. Then Proposition 4.1 implies that the unfolding $F$ is locally topologically trivial along $I \subset \Lambda$, and that $f$ and $f_{0}$ are topologically equivalent, since $I$ is connected.

Let, in general, $F=\left(f_{\lambda}, \lambda\right):\left(\mathbf{R}^{2} \times \Lambda, 0 \times \Lambda\right) \rightarrow\left(\mathbf{R}^{3} \times \Lambda, 0 \times \Lambda\right)$ be an unfolding of the form as in $\S 2$. Set $f_{\lambda}(t, x)=\left(x, B(t, \lambda)+x A(t, \lambda), B^{\prime}(t, \lambda)+x A^{\prime}(t, \lambda)\right)$. To study the set $M\left(f_{\lambda}\right)$, we set

$$
d_{\lambda}\left(t_{1}, t_{2}\right)=\left|\begin{array}{cc}
A\left(t_{2}, \lambda\right)-A\left(t_{1}, \lambda\right), & B\left(t_{2}, \lambda\right)-B\left(t_{1}, \lambda\right) \\
A^{\prime}\left(t_{2}, \lambda\right)-A^{\prime}\left(t_{1}, \lambda\right), & B^{\prime}\left(t_{2}, \lambda\right)-B^{\prime}\left(t_{1}, \lambda\right)
\end{array}\right| .
$$

Set $h_{\lambda}\left(t_{1}, t_{2}\right)=\left(t_{2}-t_{1}\right)^{-2} d_{\lambda}\left(t_{1}, t_{2}\right)$. Then $h_{\lambda}\left(t_{1}, t_{2}\right)=h_{0}\left(t_{1}, t_{2}\right)+\Psi\left(t_{1}, t_{2}, \lambda\right)$, for some $C^{\infty}$ function germ $\Psi$ of higher order than $h_{0}$ with respect to $\left(t_{1}, t_{2}\right)$. Therefore, by Lemma $3.4, h_{\lambda}^{-1}(0)$ consists of $C^{1}$ curves $t_{2}=\xi t_{1}+o\left(t_{1}\right)$, while $h_{0}^{-1}(0)$ consists of lines $t_{2}=\xi t_{1}$.

We have seen in $\S 3$, that, just for the case (iv), the root -1 of $g(z)$ does not corresponds to a locus of $M\left(f_{0}\right)$. We see that it is valid also for $f_{\lambda}$ in the case (iv), $m=1$, as follows.

Let $r$ and $s$ are both even and $m$ is odd. Set $t_{1}=t$ and $t_{2}=-t+t h, h$ being an indeterminate. Then we have

$$
A(-t+t h, \lambda)-A(t, \lambda)=-s t^{s} h-2 t^{s+1} \psi(0, \lambda)+\cdots,
$$




$$
\begin{gathered}
B(-t+t h, \lambda)-B(t, \lambda)=-2 t^{s+m}+\cdots \\
A^{\prime}(-t+t h, \lambda)-A^{\prime}(t, \lambda)=-s(r+s+m)(r+s) t^{r+s} h-2 t^{r+s+1} \tilde{\psi}(0, \lambda)+\cdots \\
B^{\prime}(-t+t h, \lambda)-B^{\prime}(t, \lambda)=-2(r+s)(s+m) t^{r+s+m}+\cdots
\end{gathered}
$$

where $\cdots$ indicates a function of higher order for $(t, h)$. Remark that

$$
\tilde{\psi}(0, \lambda)=\frac{(r+s)(r+s+m)(s+1)}{r+s+1} \psi(0, \lambda)
$$

Then

$$
d_{\lambda}(-t+t h, t)=-2 r s(r+s) t^{r+2 s+m} h+4 \frac{(r+s) r(m-1)}{r+s+1} t^{r+2 s+m+1} \psi(0, \lambda)+\cdots .
$$

Therefore, by the implicit function theorem, the equation $d_{\lambda}(-t+t h, t)=0$ has a $C^{\infty}$ solution,

$$
h(t, \lambda)=\frac{2(m-1)}{s(r+s+1)} t \psi(0, \lambda)+\cdots
$$

Now let $m=1$. Then, for any $x$ satisfying

$$
\{A(-t+t h, \lambda)-A(t, \lambda)\} x+\{B(-t+t h, \lambda)-B(t, \lambda)\}=0,
$$

we have that $|x|>C$, for some uniform positive constant $C$ for any $\lambda$ belonging to a compact set on $\Lambda$.

We observe also, in the case (iv) $m \geq 3$,

$$
x=-\frac{r+s+1}{r+s+m} t^{m-1} \frac{1}{\psi(0, \lambda)}+\cdots,
$$

provided $\psi(0, \lambda) \neq 0$.

Thus, in each of seven cases of Theorem 4.1, any branch of $M\left(f_{\lambda}\right)$ in the $(t, x)$ plane is a $C^{1}$ curve of form $x=a t^{m}+o\left(t^{m}\right)$ corresponding to the branch $x=a t^{m}$ in $M\left(f_{0}\right)$. Its relative position with $C_{0}, L$ and other branches does not change as $\lambda$ varies.

Let $C$ be a $C^{1}$ curve $x=a t^{m}+o\left(t^{m}\right)$, for some $a \in \mathbf{R}$, on the $(t, x)$-plane. Then $f_{\lambda}(C)$ has the parametrisation

$t \mapsto\left(a t^{m}+o\left(t^{m}\right),(1+a) t^{s+m}+o\left(t^{s+m}\right),\{(r+s)(s+m)+a s(r+s+m)\} t^{r+s+m}+o\left(t^{r+s+m}\right)\right)$.

Therefore the curves of form $x=a t^{m}+o\left(t^{m}\right)$ with different coefficients $a$ have disjoint images by $f_{\lambda}$. In particular, $f_{\lambda}$ has no triple points. The Nash lifting $\tilde{f}_{\lambda}$ is also perturbed by higher order terms than $\tilde{f}_{0}$. Therefore $f_{\lambda}$ has also no points of self-tangency. 
Now the non-immersive locus of $F$ is decomposed into the locus $C_{0}(F)$ of parameters of original curves, and $L(F)=\{t=0\} \times \Lambda \subset \mathbf{R}^{2} \times \Lambda$. Set $D_{0}(F)=F\left(C_{0}(F)\right), L^{\prime}(F)=$ $F(L(F))=L^{\prime} \times \Lambda$. Moreover set

$$
M(F)=\text { the closure of }\left\{(t, x, \lambda) \in\left(\mathbf{R}^{2} \times \Lambda, 0 \times \Lambda\right) \mid \# F^{-1}(F(t, x, \lambda)) \geq 2\right\},
$$

and $W(F)=F(M(F))$. Then, by the above arguments, we have

Proposition $4.2(0) F^{-1}(0 \times \Lambda)=0 \times \Lambda$.

(i) $\left(C_{0}(F) \cup L(F)\right) \cap M(F)=0 \times \Lambda, \quad\left(D_{0}(F) \cup L^{\prime}(F)\right) \cap W(F)=0 \times \Lambda$.

(ii) $C_{0}(F) \cap(L \times \Lambda)=0 \times \Lambda, D_{0}(F) \cap\left(L^{\prime} \times \Lambda\right)=0 \times \Lambda$.

(iii) $F$ has no points of self-tangency.

(iv) For $(t, x, \lambda) \in M(F)-(0 \times \Lambda), \# F^{-1}(F(t, x, \lambda))=2$.

Set, in $\mathbf{R}^{2} \times \Lambda, V_{0}=\mathbf{R}^{2} \times \Lambda-\left(C_{0}(F) \cup L(F) \cup M(F)\right), V_{1}^{1}=M(F)-(0 \times \Lambda)$, $V_{1}^{2}=C_{0}(F)-(0 \times \Lambda), V_{1}^{3}=L(F)-(0 \times \Lambda), V_{2}=0 \times \Lambda$. Further set, in $\mathbf{R}^{3} \times \Lambda$, $W_{0}=\mathbf{R}^{3} \times \Lambda-\left(F\left(\mathbf{R}^{2} \times \Lambda\right)\right), W_{1}=F\left(\mathbf{R}^{2} \times \Lambda\right)-\left(D_{0}(F) \cup L^{\prime}(F) \cup W(F)\right), W_{2}^{1}=$ $W(F)-(0 \times \Lambda), W_{2}^{2}=D_{0}(F)-(0 \times \Lambda), W_{2}^{3}=L^{\prime}(F)-(0 \times \Lambda), W_{3}=0 \times \Lambda$. Then consider the stratifications

$$
\mathbf{V}=\left\{V_{0}, V_{1}^{1}, V_{1}^{2}, V_{1}^{3}, V_{2}\right\}, \quad \mathbf{W}=\left\{W_{0}, W_{1}, W_{2}^{1}, W_{2}^{2}, W_{2}^{3}, W_{3}\right\}
$$

of $\mathbf{R}^{2} \times \Lambda$ and $\mathbf{R}^{3} \times \Lambda$ respectively. Remark that, by Proposition $4.2,(\mathbf{V}, \mathbf{W})$ are in fact a stratification of $F$, and that $F$ maps immersively $V_{0}, V_{1}^{1}, V_{1}^{2}, V_{1}^{3}, V_{2}$ to $W_{1}, W_{2}^{1}, W_{2}^{2}, W_{2}^{3}, W_{3}$ respectively. Then the following implies Proposition 4.1 and therefore the "if" part of Theorem 1.1 via the second isotopy lemma [9] :

Proposition 4.3 The stratifications $\mathrm{V}$ and $\mathrm{W}$ are Whitney regular. Moreover $(\mathrm{V}, \mathrm{W})$ is Thom regular with respect to $F$.

Proof: Whitney regularity can be directly verified for each pair of strata of $\mathbf{V}$ and $\mathbf{W}$ respectively. We here show only the least trivial step: $W_{1}$ is Whitney regular (b) over $W_{3}$.

Let $p_{i}=\left(t^{(i)}, x^{(i)}, \lambda^{(i)}\right) \in\left(\mathbf{R}^{2}-0\right) \times \Lambda$, and $q_{i}=\left(0, \mu^{(i)}\right) \in \mathbf{R}^{3} \times \Lambda$ be sequence of points, $i=1,2, \ldots$ Assume both $F\left(p_{i}\right)$ and $q_{i}$ converge to a point $\left(0, \lambda_{0}\right) \in \mathbf{R}^{3} \times \Lambda$ and the sequence of lines $\overline{F\left(p_{i}\right) q_{i}}$ converges to $\ell \subset T_{\left(0, \lambda_{0}\right)}\left(\mathbf{R}^{3} \times \Lambda\right)$. Remark that $F_{*}\left(T_{p_{i}}\left(\mathbf{R}^{2} \times \Lambda\right)\right)$ tends to $\left\{d x_{3}=0\right\} \subset T_{\left(0, \lambda_{0}\right)}\left(\mathbf{R}^{3} \times \Lambda\right)$, which is equal to the image of $\left(0, \lambda_{0}\right)$ under the Nash lifting of $F$. Therefore it suffices to show $\ell \subset\left\{d x_{3}=0\right\}$.

Suppose $\ell$ is not contained in $\left\{d x_{3}=0\right\}$. Then the ratios $x_{1} / x_{3}$ and $x_{2} / x_{3}$ of components of $F=\left(x_{1}, x_{2}, x_{3}, \lambda\right)$ would be bounded as $i \rightarrow \infty$. Assume, for an infinite number of $i$, that $\left|t^{(i)}\right| \leq 2\left|x^{(i)}\right|$. Then, from the concrete form of $F$, we see, for such $i$ and for some positive constant $c$ independent of $i$, that $\left|x_{1} / x_{3}\right| \geq c /\left|t^{(i)}\right|^{r+s} \rightarrow \infty,(i \rightarrow \infty)$. Assume 
contrarily, for an infinite number of $i$, that $\left|t^{(i)}\right| \geq 2\left|x^{(i)}\right|$. Then we see, for such $i$ and for some positive constant $d$ independent of $i$, that $\left|x_{2} / x_{3}\right| \geq d /\left|t^{(i)}\right|^{r} \rightarrow \infty,(i \rightarrow \infty)$. This leads to a contradiction.

Thom regularity is clear since the restriction of $F$ to each stratum is an immersion.

Proof of Proposition 1.4: By the arguments above, we have seen that each $f_{\lambda}$ has the properties of Proposition 1.4, except for the case (iv), $m \geq 3$.

In the case (iv), $m \geq 3$, depending on $\lambda, M\left(f_{\lambda}\right)$ has possibly one extra branch $C_{-1}$ : $x=c t^{m-i}+o\left(t^{m-i}\right)$, for some non-zero constant $c$ and for some odd integer $i, 1 \leq i \leq m$. This branch, however, does not intersects to other branches of $M\left(f_{\lambda}\right)$ outside of the origin, both in the $(t, x)$ plane and in the $\left(x_{1}, x_{2}, x_{3}\right)$ space in a neighbourhood (depending on $\left.\lambda\right)$ of the origin, by the effect of orders of curves. Furthermore we see that the self-tangency does not occur also along $C_{-1}$, since the Nash lifting separates $C_{-1}$. Moreover, in all cases, each developable is homeomorphic to the cone of one of the spherical curves shown in Fig.1.

\section{$5 \quad$ Non-determined types}

First we observe the following fact on the duality of contact at an ordinary point of plane curves (cf. [4], [25]), which is used below:

Lemma 5.1 Let $C$ and $C^{\prime}$ be plane curve-germ at a common ordinary point $p_{0}$ respectively. If $C^{\prime}$ lies in the convex side of $C$, then the dual $C^{\prime *}$ lies in the concave side of $C^{*}$.

Proof: By the projective duality in the plane, the tangent line $L$ to a point of $C^{\prime}$ intersects to a point $p$ of $C$ if and only if the tangent line $p^{*}$ to $C^{*}$ intersects to the point $L^{*} \in C^{\prime *}$.

Proof of Proposition 1.3: Let $m$ is odd, $m \geq 3, s$ is even and $r$ is even. Then, as we have seen in the previous section, an extra homeomorphism class appears, which is illustrated at the last position in each of Fig. 1,2 and 3. This occurs in fact at least if $\psi(0) \neq 0$. Therefore, in this case, we have exactly two homeomorphism classes.

Let $m, s$ and $r$ are all even. Consider, in the dual space, a curve-germ $\gamma^{*}$

$$
\left(y_{1}(t), y_{2}(t), y_{3}(t)\right)=\left(t^{r}, t^{r+s}, t^{r+s+m}+\rho(t)\right),
$$

for an infinitely flat non-negative $C^{\infty}$ function $\rho$ with $\rho(t)=0, t<0$. Moreover assume that $\rho$ is infinitely flat at each zero point of $\rho$. Remark that, if $\rho \equiv 0$, then $\gamma^{*}(t)=\gamma^{*}\left(t^{\prime}\right)$ 
if and only if $t^{\prime}=-t$. Let $\operatorname{supp}(\rho)$ denote the support of $\rho$. Then $\operatorname{supp}(\rho) \cup(-\operatorname{supp}(\rho))$ is equal to the set-germ $X_{\rho}=\left\{t \in(\mathbf{R}, 0) \mid\left(\gamma^{*}\right)^{-1}\left(\gamma^{*}(t)\right) \neq\{t\}, t \geq 0\right\}$. The dual curve $\gamma$ of $\gamma^{* *}$ is of type $(m, m+s, m+s+r)$. For the parametrisation $f_{\rho}$ of the tangent developable of $\gamma$, we see, from the projective duality, that the germ $X_{p} \times \mathbf{R}$ is equal to the set of self-tangency:

$$
\left\{(t, x) \in\left(\mathbf{R}^{2}, 0\right) \mid f(t, x)=f\left(t^{\prime}, x\right), f_{*} T_{(t, x)} \mathbf{R}^{2}=f_{*} T_{\left(t^{\prime}, x\right)} \mathbf{R}^{2} \text {, for some } t \neq t^{\prime}\right\} .
$$

Take a section of the developable, parametrised by $f_{\rho}$, by the plane $\left\{x_{1}=c\right\}$. This plane curve $C=\left.f_{\rho}\right|_{\{x=c\}}$ is dual to the projection $C^{*}: t \mapsto\left(y_{1}(t), c y_{2}(t)+y_{3}(t)\right)$ from the point $[0,0,1,-c] \in \mathbf{R} P^{3 *}$ of $\gamma^{*}$. Therefore the section $C$ has the same set of self-tangency that $C^{*}$ has, and is equal to $X_{\rho}$. Therefore, by Lemma 5.1, we see, for $(t, x),\left(t^{\prime}, x\right) \in X_{\rho} \times \mathbf{R}$ with $t \neq t^{\prime}$, that the multi-germ of $f_{\rho}$ along $\left\{(t, x),\left(t^{\prime}, x\right)\right\}$ is never topologically equivalent to any transverse multi-germ.

Now, if $f_{\rho}$ is topologically equivalent to another $f_{\rho^{\prime}}$, then the set-germ $\left(\mathbf{R}^{2}, X_{\rho} \times \mathbf{R}, 0\right)$ is relatively homeomorphic to $\left(\mathbf{R}^{2}, X_{\rho^{\prime}} \times \mathbf{R}, 0\right)$. Notice that any germ of closed subset of $\left(\mathbf{R}_{\geq 0}, 0\right) \subset(\mathbf{R}, 0)$ can be obtained as the support of some $\rho$ satisfying the above conditions. Thus we see the existence of uncountably many homeomorphism classes of the tangent developables of curves of type $(m, m+s, m+s+r)$.

\section{References}

[1] V.I. Arnold, Singularities of Caustics and Wave Fronts, Kluwer Academic Publishers, Dordrecht, 1990.

[2] V.I. Arnold, Topological Invariants of Plane Curves and Caustics, University Lecture Series 5, Amer. Math. Soc., Providence, 1994.

[3] J.J. nuño Ballesteros, On the number of the triple points of the tangent developable, Geom. Dedicata 47-3 (1993), 241-254.

[4] J.W. Bruce, On contact of hypersurfaces, Bull. London. Math. Soc. 13 (1981), 51-54.

[5] J.W. Bruce, P.J. Giblin, Curves and Singularities, 2nd. ed., Cambridge Univ. Press, Cambridge, 1992.

[6] A. Cayley, On the developable surfaces which arise from two surfaces of second order, Cambridge and Dublin Math. J. 2 (1850), 46-57 = The Collected Mathematical Papers vol.I, pp. $486-495$.

[7] J.P. Cleave, The form of the tangent developable at points of zero torsion on space curves, Math. Proc. Camb. Phil. Soc. 88 (1980), 403-407.

[8] T. Gaffney, A. du Plessis, More on the determinacy of smooth map-germs, Invent. Math. 66 (1982), 137-163. 
[9] C.G. Gibson, K. Wirthmüller, A.A. du Plessis, E.J.N. Looijenga, Topological Stability of Smooth Mappings, Lecture Notes in Math., 552, Springer-Verlag, 1976.

[10] A.B. Givental, Lagrangian imbeddings of surfaces and unfolded Whitney umbrella, Funksional Anal. i Prilozhen., 20-3 (1986), 35-41.

[11] G. Ishikawa, Determinacy of envelope of the osculating hyperplanes to a curve, Bull. London Math. Soc., 25 (1993), 603-610.

[12] G. Ishikawa, Parametrized Legendre and Lagrange varieties, Kodai Math. J., 17-3 (1994), $442-451$.

[13] G. Ishikawa, Developable of a curve and its determinacy relatively to the osculation-type, Quarterly J. Math., 46 (1995), 437-451.

[14] S. Janeczko, M. Roberts, Classifications of symmetric caustics I: symplectic equivalence, Singularity theory and its application II, ed. by M. Roberts, I. Stewart, Lecture Notes in Math. 1463, Springer-Verlag (1991), pp. 193-219.

[15] A.G. Khovanskil, Fewnomials, Transl. Math. Monographs 88, Amer. Math. Soc., Providence, 1991.

[16] N.H. Kuiper, $C^{1}$-equivalence of functions near isolated critical points, Symposium in Infinite Dimensional Topology, (Baton Rouge, 1967).

[17] T.C. Kuo, On $C^{0}$ sufficiency of functions near isolated critical points, Topology, 8 (1969), $167-171$.

[18] D. Mond, On the tangent developable of a space curve, Math. Proc. Camb. Phil. Soc. 91 (1982), 351-355.

[19] D. Mond, Singularities of the tangent developable surface of a space curve, Quart. J. Math. Oxford, 40 (1989), 79-91.

[20] I.R. Porteous, Geometric Differentiation, for the Intelligence of Curves and Surfaces, Cambridge Univ. Press, Cambridge, 1994.

[21] O.P. Shcherbak, Projectively dual space curves and Legendre singularities, Trudy Tbiliss. Univ. 232-233 (1982), 280-336. = Sel. Math. Sov. 5-4 (1986), 391-421.

[22] O.P. Shcherbak, Wavefront and reflection groups, Russian Math. Surveys 43-3 (1988), 149-194.

[23] J.J. Stoker, Differential Geometry, Pure and Applied Math. 20, Wiley-Interscience, New York 1969.

[24] C.T.C. Wall, Finite determinacy of smooth map-germs, Bull. London Math. Soc., 13 (1981), 481-539.

[25] C.T.C. Wall, Duality of singular plane curves, J. London Math. Soc., 50 (1994), 265-275. 


\section{Goo ISHIKAWA}

Department of Mathematics, Hokkaido University, Sapporo 060, JAPAN

e-mail: ishikawa@math.hokudai.ac.jp 
Figure 1.

(i)


Figure 2.

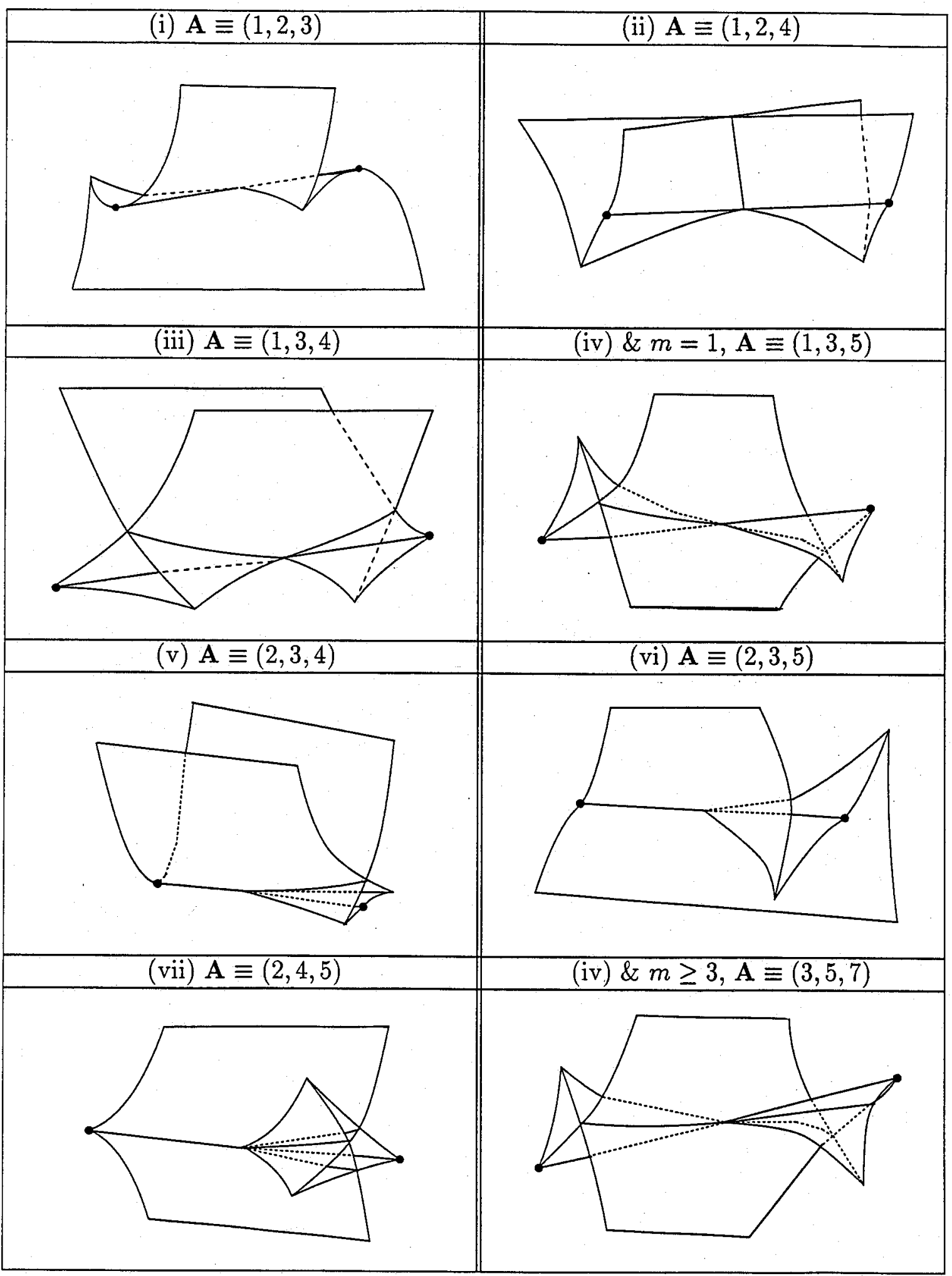


Figure 3.



